\title{
ACHATINA FULICA MUCUS ATTENUATES ULTRAVIOLET B-INDUCED FIBROBLAST PHOTOAGING THROUGH REDUCING INFLAMMATION, ANGIOGENESIS, AND MATRIX METALLOPROTEINASE
}

\author{
CH. TRI NURYANA ${ }^{1 *}$, SOFIA MUBARIKA ${ }^{2}$, YOHANES WIDODO WIROHADIDJOJO ${ }^{3}$, NUR ARFIAN ${ }^{4}$, \\ PUTU MEGA ADITYA DEVI AYU MARA ${ }^{5}$, AHMAD FAIZ HUWAIDI ${ }^{5}$
}

${ }^{1}$ Doctoral Program of Medicine and Health, Faculty of Medicine, Public Health and Nursing, Universitas Gadjah Mada, Yogyakarta, Indonesia. ${ }^{2}$ Department of Histology and Cell Biology, Faculty of Medicine, Public Health and Nursing, Universitas Gadjah Mada, Yogyakarta, Indonesia. ${ }^{3}$ Department of Dermatology and Venereology, Faculty of Medicine, Public Health and Nursing, Universitas Gadjah Mada, Yogyakarta, Indonesia. ${ }^{4}$ Department of Anatomy, Faculty of Medicine, Public Health and Nursing, Universitas Gadjah Mada, Yogyakarta, Indonesia. ${ }^{5}$ Undergraduate Program of Medicine and Health, Faculty of Medicine, Public Health and Nursing, Universitas Gadjah Mada, Yogyakarta, Indonesia. Email: ch_trinuryana@ugm.ac.id

Received: 27 February 2020, Revised and Accepted: 02 April 2020

\section{ABSTARCT}

Objective: This study aimed to observe the effects of Achatina fulica mucus (AFM) on ultraviolet B (UVB)-induced fibroblast photoaging by assessing monocyte chemotactic protein (MCP)-1, vascular endothelial growth factor (VEGF), matrix metalloproteinases (MMP)-3, and MMP-12 mRNA expressions

Methods: Cell cultures of normal human dermal fibroblasts (NHDFs) were divided into six groups: Group 1 was normal fibroblasts without UVB irradiation as normal control and Groups 2-5 consisted of $100 \mathrm{~mJ} / \mathrm{cm}^{2}$ UVB-induced aged fibroblasts. Group 2 had no treatment as negative control, Group 3 was treated by platelet-rich plasma $10 \%$ as positive control group, and Groups 4-6 were treated by various concentrations of AFM (3.9 $\mu$, $15.625 \mu \mathrm{l}$, and $62.5 \mu \mathrm{l}$ ). The MCP-1, VEGF, MMP-3, and MMP-12 mRNA expressions in the different NHDF groups were assessed by quantitative polymerase chain reaction.

Results: The mRNA expressions of MCP-1, VEGF, MMP-3, and MMP-12 in the AFM group compared to the UVB group decreased 8, 5, 5, and 4 folds, respectively. AF62 exhibited the highest improvement among the other AFM-treated groups.

Conclusion: AFM treatment attenuates UVB-induced fibroblasts photoaging by reducing inflammation, angiogenesis, and matrix metalloproteinases.

Keywords: Ultraviolet B, Photoaging, Achatina fulica, Matrix metalloproteinase, Inflammation, Angiogenesis.

(c) 2020 The Authors. Published by Innovare Academic Sciences Pvt Ltd. This is an open access article under the CC BY license (http://creativecommons. org/licenses/by/4. 0/) DOI: http://dx.doi.org/10.22159/ajpcr.2020.v13i5.37284

\section{INTRODUCTION}

The main external factor for skin aging is ultraviolet (UV) radiation, particularly UVB (290-320 nm). UVB has the most energy compared to UVA (320-400 nm) and UVC (200-290 nm) that penetrate into the epidermis and dermis [1]. UVB is responsible for skin damage called photoaging which is represented by crinkle, lose elasticity, frailty, rough skin texture, and teleangiectasies [2]. This condition occurs because UVB triggers inflammation and degradation of the extracellular matrix such as collagen and elastin [3].

UVB generates inflammation in the skin by transcription and releasing of monocyte chemotactic protein (MCP)-1. MCP-1 is the most prominent chemokines of the senescence-associated secretory phenotype (SASP) that expressed and released by fibroblasts and endothelial cells, leading to inflammation and vascular changes in the skin. This vascular changes managed by angiogenic factors as well as vascular endothelial growth factor (VEGF) [4]. In chemokine production and inflammatory response induction, reactive oxygen species (ROS) is known to be an important mediator. Inflammation activates various matrix metalloproteinases (MMP) degradation matrices, which leads to abnormal extra cellular matrix including changes in collagen and elastin. The MMP responsible for collagen degradation is MMP-3 while for elastin degradation, it is MMP-12 [5].

Recent research has focused on discovering anti-aging agents from natural ingredients, plant products, or herbal extracts [6]. Achatina fulica mucus (AFM) is a natural remedy containing anti-aging agents that have been used in traditional medicine. The previous study revealed that AFM contains Achasin, a broad-spectrum antibiotic. Moreover, AFM also contains acharan sulfate which is a glycosaminoglycan, antiinflammation, antiangiogenic, copper which contributes to collagen synthesis, and Vitamins A and $\mathrm{E}$ as antioxidants $[7,8]$.

Accordingly, the present experiment was aimed to determine how the AFM, which is rich in antioxidants, anti-inflammation activity, and antiangiogenic can be turned into high end-value products. This study also observes the effect of AFM in the inhibition of MMP on UVBinduced fibroblasts photoaging through the reduction of inflammation and angiogenesis.

\section{METHODS}

This in vitro experimental study had previously obtained approval from the ethics committee of the Faculty of Medicine, Public Health and Nursing, Universitas Gadjah Mada (Ref: KE/FK/0682/EC/2018).

\section{Snails mucus and materials}

AFM was collected by triggering the mucus secretion using 5-10 $\mathrm{V}$ electricity shock for 30-60 s. The mucus was stored in a sterilized jar before it was converted into powder by the freeze-drying process. The platelet-rich plasma (PRP) was purchased from the DermatoVenereology Department of the Faculty of Medicine, Public Health and Nursing, Universitas Gadjah Mada. 
Cell culture

Human dermal fibroblasts at passages $>4$ from 11 to 13 years old boy's foreskins were obtained from the Dermato-Venereology Department of Medicine, Public Health and Nursing Faculty, Universitas Gadjah Mada and maintained in Dulbecco's Modified Eagle's Medium (DMEM; SigmaAldrich Corporation, St. Louis, MO, USA) supplemented with $10 \%$ heat in activated FBS, 100 units $/ \mathrm{ml}$ penicillin, and $100 \mu \mathrm{g} / \mathrm{ml}$ streptomycin (Pen-Strep-Gibco; Invitrogen Corporation, Carlsbad, CA, USA) in a humidified atmosphere of $5 \% \mathrm{CO}_{2}$ at $37^{\circ} \mathrm{C}$. For subculture, the medium was eliminated and cells were rinsed with phosphate buffer saline (PBS). Then, the cells were detached using trypsin and were cultured with completely fresh growth medium.

\section{UVB irradiation and treatments}

The cells were plated until they had attained $80-90 \%$ confluence, rinsed with PBS and then exposed to UVB light in fresh PBS-filled wells. Exposure to UVB was performed using a Spectrolinker XL-1500 UV crosslinker (Spectronics Corporation, Westbury, NY, USA) which emits the majority of its energy within the UVB range $(280-320 \mathrm{~nm})$ peaking at $312 \mathrm{~nm}$. The intensity of UVB radiation was measured using a phototherapy radiometer (International Light Technologies, Newburyport, MA, USA). The cells were exposed to UVB radiation at a dose of $100 \mathrm{~mJ} / \mathrm{cm}^{2}$ for $330 \mathrm{~s}$. After UVB irradiation, the cells were washed with PBS 3 times. The cells were immediately treated with AFM in $3.9 \mu \mathrm{L}, 15.625 \mu \mathrm{L}$, and $62.5 \mu \mathrm{L}$ concentrations. The cells received no pre-treatment and were not exposed to UVB irradiation served as normal controls (NCs). The cells that were exposed to UVB radiation without any pretreatment were used as negative controls. The cells that were exposed to UVB radiation with $10 \%$ PRP treatment were used as positive control. Then, the cells were incubated for $72 \mathrm{~h}$ and after that cell viability was measured using MTT assay.

\section{Measurement of MCP-1, VEGF, MMP-3, and MMP-12 mRNA expressions}

Quantitative real-time polymerase chain reaction (qRT-PCR) was conducted to measure the expression levels of MMP-3 and MMP-12. The mRNA expression levels were normalized to GAPDH. RNA was extracted from the cells by a miRCURY cell and plant isolation kit (Exiqon; Lot\# 32213). cDNA was synthesized using miRCURY LNA Universal RT microRNA PCR Universal cDNA Synthesis Kit II, 8-64 rxns (Exiqon; Lot \# 629693). qRT-PCR was conducted using protocol from Applied Biosystem 7500 FAST real-time PCR using Toyobo Thunderbird $\AA$ SYBR $^{\circledR}$ qPCR Mix (cat\#QPS-201). The thermocycling conditions were as follows: Initial denaturation at $95^{\circ} \mathrm{C}$ for $2 \mathrm{~min}$, then denaturation at $95^{\circ} \mathrm{C}$ for $10 \mathrm{~s}$, annealing at $58^{\circ} \mathrm{C}$ for $15 \mathrm{~s}$, and extension at $72^{\circ} \mathrm{C}$ for $20 \mathrm{~s}$, for a total of 40 cycles. GAPDH served as an internal reference. The expression levels of MCP-1, VEGF, MMP-3, and MMP-12 mRNA were calculated using the $2-\triangle \Delta \mathrm{Cq}$ method. The following primers were used: MCP-1, forward TCCCAAAGAAGCTGTGATCTTCA and reverse TTTGCTTGTCCAGGTGGTCC; VEGF, forward CGCTCGGTGCTGGAATTTG and reverse TGGGGAATGGCAAGCAAAA; MMP-3, forward GACAAAGGATACAACAGGGACCA and reverse ACCGAGTCAGGTCTGTGAG; and MMP-12, forward GATGCTGTCACTACCGTGGGAA and reverse CAATGCCAGATGGCAAGGTTGG. Each experiment was performed in triplicate.

\section{Statistical analysis}

All data were presented as mean \pm standard deviation. Significant differences between groups were analyzed by ANOVA followed by the post hoc test. $\mathrm{p}<0.05$ was considered to represent statistical significance.

\section{RESULTS}

\section{AFM downregulated MCP-1 mRNA expression}

UV exposure increases MCP-1 which is one of the most well-known chemokines of the SASP that plays an important role in the inflammatory response. In humans, this enzyme is encoded by the MCP-1 gene. To observe the effects of AFM on UVB-mediated MCP-1 mRNA expression, the mRNA levels were quantified by qRT-PCR. After UVB irradiation, the NDHFs were incubated for $72 \mathrm{~h}$ with $3.9,15.625$, and $62.5 \mu \mathrm{g} / \mathrm{mL}$ concentrations of AFM. As shown in Fig. 1, UVB induction in the UVB group demonstrated significantly higher MCP-1 mRNA expression compared to the NC group. Significantly lower mRNA expressions of MCP-1 were shown in the PRP and AFM-treated groups. In the AFM group, we found significant differences between AF62 compared to $\mathrm{AF} 3$ and AF15, but there was no significant difference between AF3 and AF15.

\section{AFM downregulated VEGF mRNA expression}

UV exposure increases angiogenesis which is regulated by VEGF. To observe the effects of AFM on UVB-mediated VEGF mRNA expression, the mRNA levels were quantified by qRT-PCR. After UVB irradiation, the NDHFs were incubated for $72 \mathrm{~h}$ with $3.9,15.625$, and $62.5 \mu \mathrm{g} / \mathrm{mL}$ concentrations of AFM. As shown in Fig. 2, UVB induction in the UVB group demonstrated significantly higher VEGF-1 mRNA expression compared to the NC group. Significantly lower mRNA expressions of VEGF were shown in the PRP and AFM-treated groups. In the AFM group, we found significant differences between AF62 compared to AF3 and $\mathrm{AF} 15$, and vice versa.

\section{AFM downregulated MMP-3 mRNA expression}

UV exposure increases MMP-3 mRNA expression which triggers degradation of collagen, especially type III collagen in the dermis. To observe the effects of AFM on UVB-mediated mRNA expression of MMP-3, the mRNA levels were quantified by qRT-PCR. After UVB irradiation, the NDHFs were incubated for $72 \mathrm{~h}$ with $3.9,15.625$, and $62.5 \mu \mathrm{g} / \mathrm{mL}$ concentrations of AFM. As shown in Fig. 3, UVB induction in the UVB group demonstrated significantly higher MMP-3 mRNA expression compared to the NC group. Significantly lower mRNA expressions of MMP-3 were shown in the PRP and AFM-treated groups. In the AFM group, we found significant differences between AF62 compared to AF3 and AF15, but there was no significant difference between AF3 and AF15.

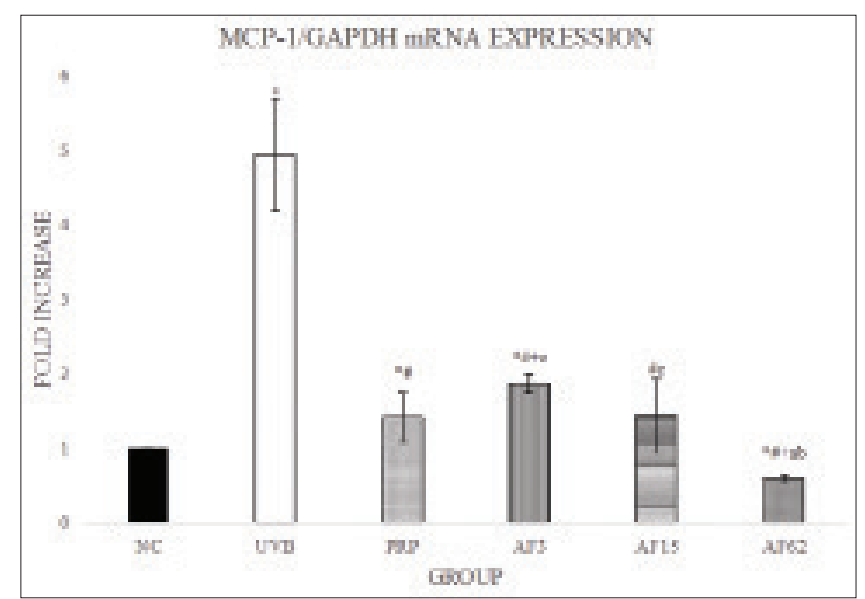

Fig. 1: Quantitative polymerase chain reaction examination of vascular endothelial growth factor expression. Normal human dermal fibroblasts were induced with ultraviolet $B$ (UVB) $100 \mathrm{~mJ} / \mathrm{cm}^{2}$ and administered with various concentrations of Achatina fulica mucus (AFM) $(3.9 \mu \mathrm{l}, 15.625 \mu \mathrm{l}$, and $62.5 \mu \mathrm{l})$. The values are provided as the mean $\pm S E M . * p<0.05$ indicates significant difference versus the normal control (NC). ${ }^{*} p<0.05$ indicates significant difference versus UVB as negative control. $+p<0.05$ indicates significant difference versus platelet-rich plasma (PRP) as positive control. In different concentrations of AFM groups, $a, b$, and $c, p<0.05$ indicates significant difference versus AF3, AF15, and AF62, respectively. $\mathrm{NC}$ is the NDHFs not exposed to UVB irradiation and received no treatment, UVB is the NDHFs exposed to UVB irradiation and received no treatment,

PRP is the NDHFs exposed to UVB irradiation and received treatment with PRP $10 \%$, while AFM is the NDHFs exposed to UVB irradiation and received treatment with various concentrations (3.9 $\mu \mathrm{l}, 15.625 \mu \mathrm{l}$, and $62.5 \mu \mathrm{l})$ 


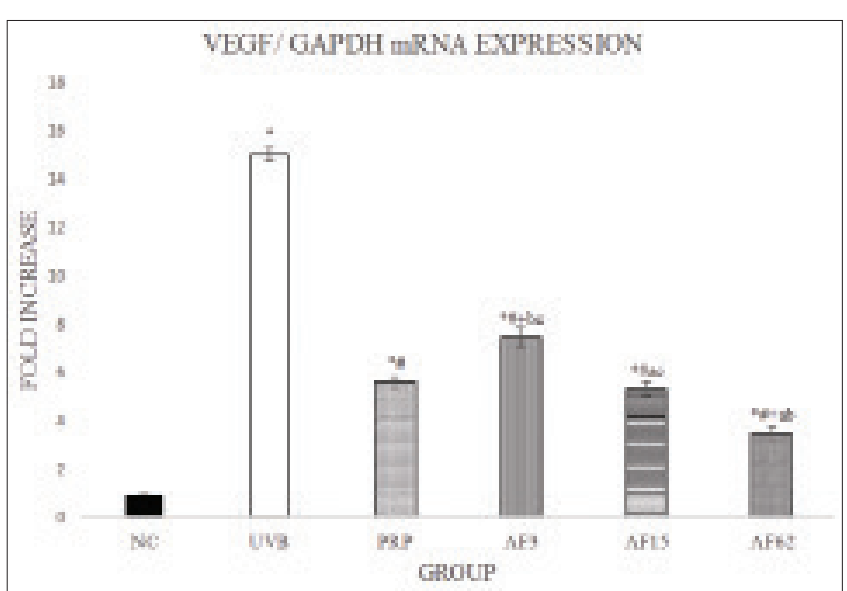

Fig. 2: Quantitative polymerase chain reaction analysis of vascular endothelial growth factor expression. Normal human dermal fibroblasts were induced with ultraviolet B (UVB) $100 \mathrm{~mJ} / \mathrm{cm}^{2}$ and administered with various concentrations of Achatina fulica mucus (AFM) $(3.9 \mu \mathrm{l}, 15.625 \mu \mathrm{l}$, and $62.5 \mu \mathrm{l})$. The values are provided as the mean \pm SEM. ${ }^{*}$ p $<0.05$ indicates significant difference versus the normal control (NC). ${ }^{*} \mathbf{p}<\mathbf{0 . 0 5}$ indicates significant difference versus UVB as negative control. $+\mathbf{p}<0.05$ indicates significant difference versus platelet-rich plasma (PRP) as positive control. In different concentrations of AFM groups, a, b, and c, p<0.05 indicates

significant difference versus AF3, AF15, and AF62, respectively

NC is the NDHFs not exposed to UVB irradiation and received no treatment, UVB is the NDHFs exposed to UVB irradiation and received no treatment, $P R P$ is the NDHFs exposed to UVB irradiation and received treatment with PRP 10\%, while AFM is the NDHFs exposed to UVB irradiation and received treatment with various concentrations $(3.9 \mu \mathrm{l}, 15.625 \mu \mathrm{l}$, and $62.5 \mu \mathrm{l})$

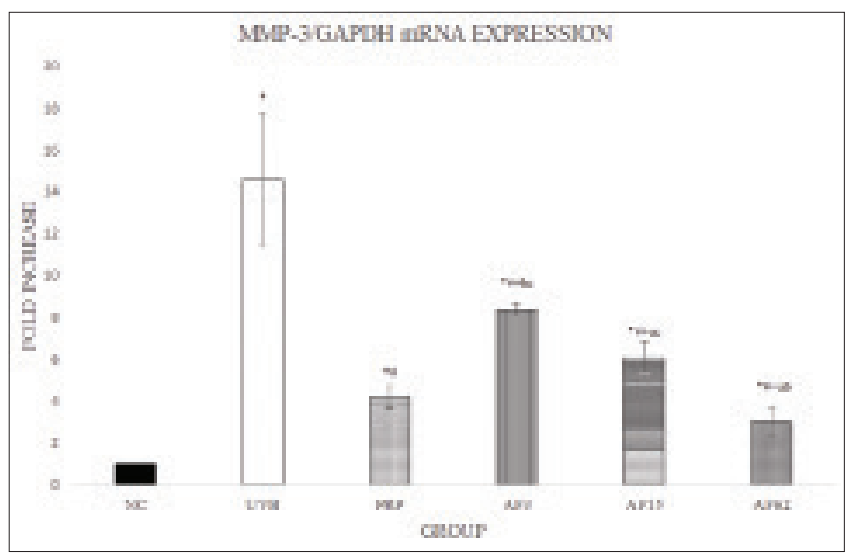

Fig. 3: Quantitative polymerase chain reaction interpretation of matrix metalloproteinases-3 mRNA expression. Normal human dermal fibroblasts were induced with ultraviolet B (UVB) $100 \mathrm{~mJ} / \mathrm{cm}^{2}$ and administered with various concentrations of Achatina fulica mucus (AFM) $(3.9 \mu \mathrm{l}, 15.625 \mu \mathrm{l}$, and $62.5 \mu \mathrm{l})$. The values are provided as the mean \pm SEM. ${ }^{*} \mathbf{p}<0.05$ indicates significant difference versus the normal control (NC). ${ }^{\#} \mathbf{p}<0.05$ indicates significant difference versus UVB as negative control. $+\mathrm{p}<0.05$ indicates significant difference versus platelet-rich plasma (PRP) as positive control. In different concentrations of AFM groups, a, b, and $c, p<0.05$ indicates significant difference versus AF3, AF15, and AF62, respectively. NC is the NDHFs not exposed to UVB irradiation and received no treatment, UVB is the NDHFs exposed to UVB irradiation and received no treatment, PRP is the NDHFs exposed to UVB irradiation and received treatment with PRP $10 \%$, while AFM is the NDHFs exposed to UVB irradiation and received treatment with various concentrations ( $3.9 \mu \mathrm{l}, 15.625 \mu \mathrm{l}$, and $62.5 \mu \mathrm{l})$
AFM downregulated MMP-12 mRNA expression

UV exposure increases MMP-12 mRNA expression which triggers degradation of elastin in epidermis. To observe the effects of AFM on UVB-mediated mRNA expression of MMP-12, the mRNA levels were quantified by qRT-PCR. After UVB irradiation, the NDHFs were incubated for $72 \mathrm{~h}$ with $3.9,15.625$, and $62.5 \mu \mathrm{g} / \mathrm{mL}$ concentrations of AFM. As shown in Fig. 4, UVB induction in the UVB group demonstrated significantly higher MMP-12 mRNA expression compared to the NC group. Significantly lower mRNA expressions of MMP-12 were shown in the PRP and AFM-treated groups. In the AFM group, we found significant differences between AF62 compared to AF3 and AF15, but there was no significant difference between AF3 and AF15.

\section{DISCUSSION}

This study revealed the molecular mechanism of AFM attenuates UVB-induced fibroblast photoaging through reducing inflammation, angiogenesis, and matrix metalloproteinase. The photoaging mechanisms are predominantly the effect of UVB radiation. UVBinduced ROS is a very reactive molecule that contributes to cell damage. ROS molecular signaling plays an important role in the UVBinduced inflammatory response, angiogenesis, and extracellular matrix (ECM) remodeling [5]. As a result of UVB exposure, ROS triggers skin fibroblasts to produce oxidative free radicals resulting in an imbalance of oxidants and antioxidants in the skin. Antioxidants such as Vitamins $\mathrm{A}$ and $\mathrm{E}$ are needed as effective agents for scavenging UVB-induced ROS. AFM contains $\mathrm{Cu}$, Vitamin A, and Vitamin $\mathrm{E}$ which act as powerful antioxidants $[8,9]$.

Increasing of ROS induced by UVB activates mitogen-activated protein kinase that initiates activator protein 1 (AP-1) and NF-kB. AP-1 and NF-kB regulate pro-inflammatory components such as MCP-1. MCP-1 consists of 76 amino acids which are members of the CC chemokine and play an important role in the inflammatory response [10]. To

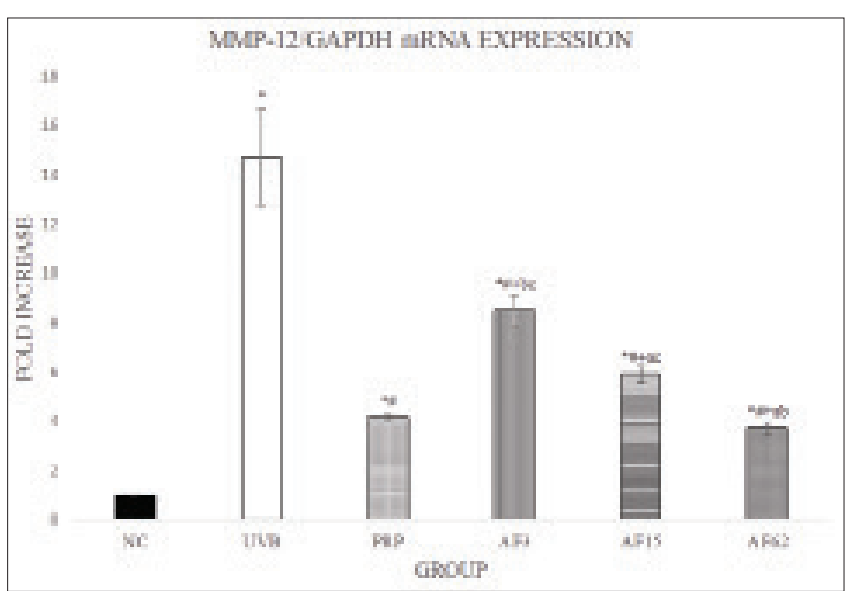

Fig. 4: Quantitative polymerase chain reaction examination of matrix metalloproteinases-12 expression. Normal human dermal fibroblasts were induced with ultraviolet B (UVB) $100 \mathrm{~mJ} / \mathrm{cm}^{2}$ and administered with various concentrations of Achatina fulica mucus (AFM) $(3.9 \mu \mathrm{l}, 15.625 \mu \mathrm{l}$, and $62.5 \mu \mathrm{l})$. The values are provided as the mean \pm SEM. ${ }^{*} \mathbf{p}<0.05$ indicates significant difference versus the normal control (NC). ${ }^{\#} \mathbf{p}<0.05$ indicates significant difference versus UVB as negative control. $+p<0.05$ indicates significant difference versus platelet-rich plasma (PRP) as positive control. In different concentrations of AFM groups, a, b, and $c, p<0.05$ indicates the significant versus AF3, AF15, and AF62, respectively. NC is the NDHFs not exposed to UVB irradiation and received no treatment, UVB is the NDHFs exposed to UVB irradiation and received no treatment, PRP is the NDHFs exposed to UVB irradiation and received treatment with PRP 10\%, while AFM is the NDHFs exposed to UVB irradiation and received treatment with various concentrations ( $3.9 \mu \mathrm{l}, 15.625 \mu \mathrm{l}$, and $62.5 \mu \mathrm{l})$ 
confirm the effect of AFM on UVB-induced inflammation, we observed the alteration of chemokine expression. The most predominant chemokines in UVB-induced skin inflammation is MCP-1. The previous research stated that MCP-1 increased in UVB-induced photoaging. This statement appropriates with our study. We revealed upregulation of MCP-1 mRNA expression after UVB exposure (Fig. 1); meanwhile, AFM treatment downregulates the MCP-1 mRNA expression. Compared to PRP as a positive control, AFM has higher effects in downregulation of MCP-1. The previous study reported that PRP is a potent antiaging material capable of inhibiting MCP-1 and enhancing collagen synthesis [11].

The previous study demonstrated that there are three sequential and overlapping events taking place in UVB-induced inflammation. The first is the early vasodilatory phase, where prostaglandin $E_{2}$ and nitric oxide are known to be involved in the induction of vascular dilatation. The second is the inflammatory phase, where various types of cells and mediators participate in the development of the erythematous changes; and (3) the last is the regressive phase consisting of an anti-inflammatory event, where several mediators and cells have been demonstrated to be involved. Angiogenesis also happens at the inflammation site and it has been shown that MMPs organize the bioavailability of angiogenic factors such as VEGF [12]

Angiogenesis is the process of new blood vessels formation. This process comprises increasing permeability of microvascular, ECM degradation, proliferation, and migration of endothelial cells [13]. The previous research stated that VEGF has been established to be generated after UVB exposure. Endothelial cells migrate out of the vessel lumen and simultaneously with the endothelial cells precursor form tubes, which sprouts from old capillaries. Endothelial cells multiply, in response to VEGF stimulating and branch into farther vessel tubes, which grows into the remodeling ECM. After new lumen is established, blood flow can start, and mature endothelial cell tubes molding new basement membranes [14]. This study revealed upregulation of VEGF mRNA expression after UVB exposure (Fig. 2), while AFM treatment downregulates the VEGF mRNA expression. Compared to PRP as a positive control, AFM has higher effects in downregulation of VEGF. This result is consistent with previous observation that found acharan sulfate possess an anti-angiogenic action. The anti-angiogenesis effect of acharan sulfate is more predominant than its anti-inflammatory effect [15].

Inflammation activates various MMPs, which leading ECM degradation including MMP-3 and MMP-12. MMP-3 or stromelysin-1 is an enzyme that degrades types III collagen, proteoglycans, fibronectin, laminin, and elastin. This enzyme has molecular weight of $54 \mathrm{kDa}$. In humans, this enzyme is encoded by the MMP-3 gene. The MMP-3 gene is part of the MMP gene cluster that is localized on chromosome 11q22.3. Therefore, an increase in MMP-3 causes the accumulation of collagen fragments, compromising the structure and function of the ECM [16]. Our study revealed upregulation of MMP-3 mRNA expression after UVB exposure (Fig. 3)' meanwhile, AFM treatment downregulates the MMP-3 mRNA expression. Compared to PRP as a positive control, AFM has higher effects in downregulation of MMP-3. As stated above, the previous study reported that PRP is a potent anti-aging material capable of inhibiting MMP-3 mRNA expression and enhancing collagen synthesis.

Macrophage elastase (MMP-12) is consistently found in elastin alteration of photoaging, indicating that it is involved in elastin remodeling. MMP12 is the main enzyme for the elastin degradation. Elastin also plays an important role to support vascular trees. This elastase cleaves the elastin and causes modification in the thickness, elasticity, and stiffness of the walls of the circulation vessels as we age [17]. UVB exposure induces MMP-12 expression, which then decreases elastin, contributing to the loss of skin elasticity [18]. The loss of elasticity or sagging has been shown in increasing human MMP-12 mRNA levels of normal human dermal fibroblast cells irradiated with UVB, while decreased in AFM various concentrations $(3.9 \mu \mathrm{l}, 15.625 \mu \mathrm{l}$, and $62.5 \mu \mathrm{l})$.

\section{CONCLUSION}

Here, we demonstrate that AFM attenuates UVB-induced fibroblast photoaging through reducing inflammation, angiogenesis, and MMP.

\section{ACKNOWLEDGMENTS}

The authors would like to express the deepest gratitude to Wiwit Ananda W.S, S.Keb. M.Sc for technical support of RT-PCR and Zulty Ananda, A.Md.A.K for technical support of in vitro study.

\section{AUTHORS' CONTRIBUTIONS}

Ch. Tri Nuryana contributed to the concept, design, statistical analysis, manuscript preparation, manuscript editing, and manuscript review. Sofia Mubarika, Yohanes Widodo Wirohadidjojo, and Nur Arfian supervised the manuscript preparation, manuscript review, and data analysis. Putu Mega Aditya Devi Ayu Mara and Ahmad Faiz Huwaidi conceived a literature search, data acquisition, and data analysis.

\section{CONFLICTS OF INTEREST}

There are no conflicts of interest.

\section{AUTHORS' FUNDING}

This work was funded by a grant from Ministry of Research, Technology and Higher Education of the Republic of Indonesia through Beasiswa Unggulan Dosen Indonesia Dalam Negeri (BUDI-DN) for the Ph.D. scholarship program (number PRJ-1375/LPDP.4/2016) and Dana Masyarakat FK-KMK Universitas Gadjah Mada 2017.

\section{REFERENCES}

1. Amaro-Ortiz A, Yan B, D'Orazio J. Ultraviolet radiation, aging and the skin: Prevention of damage by topical cAMP manipulation. Molecules 2014;19:620-19.

2. Gilchrest BA. Photoaging. J Invest Dermatol 2013;133:E2-6.

3. Scharffetter-Kochanek K, Brenneisen P, Wenk J, Herrmann G, Ma W, Kuhr L, et al. Photoaging of the skin from phenotype to mechanisms. Exp Gerontol 2000;35:307-16.

4. Cho S, Shin MH, Kim YK, Seo JE, Lee YM, Park CH, et al. Effects of infrared radiation and heat on human skin aging in vivo. J Investig Dermatol Symp Proc 2009; 14:15-9.

5. Cavinato M, Jansen-Dürr P. Molecular mechanisms of UVB-induced senescence of dermal fibroblasts and its relevance for photoaging of the human skin. Exp Gerontol 2017;94:78-82.

6. Ramdan RD, Sunendar B, Hermawan H. Naturally derived biomaterials and its processing. In: Mahyudin F, Hermawan H, editors. Biomaterials and Medical Devices. Vol. 58. Cham: Springer International Publishing; 2016. p. 23-39.

7. de la Secreción P. Assessment of antimicrobial activity and healing potential of mucous secretion of Achatina fulica. Int $\mathrm{J}$ Morphol 2012;30:365-73

8. Borkow G. Using copper to improve the well-being of the skin. Curr Chem Biol 2014;8:89-102.

9. Raman K, Arungundram S, Kuberan B. Chemogenesis of an antiangiogenic glycosaminoglycan. ACS Med Chem Lett 2014;5:644-6.

10. Kang JS, Kim HN, Kim JE, Mun GH, Kim YS, Cho D, et al. Regulation of UVB-induced IL- 8 and MCP-1 production in skin keratinocytes by increasing Vitamin C uptake via the redistribution of SVCT-1 from the cytosol to the membrane. J Invest Dermatol 2007;127:698-706.

11. Cho JM, Lee YH, Baek RM, Lee SW. Effect of platelet-rich plasma on ultraviolet b-induced skin wrinkles in nude mice. J Plast Reconstr Aesthet Surg 2011;64:e31-9.

12. Richarz NA, Boada A, Carrascosa JM. Angiogenesis in dermatologyinsights of molecular mechanisms and latest developments. Actas Dermosifiliogr 2017; 108:515-23.

13. Hirakawa S, Fujii S, Kajiya K, Yano K, Detmar M. Vascular endothelial growth factor promotes sensitivity to ultraviolet B-induced cutaneous photodamage. Blood 2005; 105:2392-9.

14. Yano K, Oura H, Detmar M. Targeted overexpression of the angiogenesis inhibitor thrombospondin-1 in the epidermis of transgenic mice prevents ultraviolet-b-induced angiogenesis and cutaneous photodamage. J Invest Dermatol 2002;118:800-5

15. Ghosh AK, Hirasawa N, Lee YS, Kim YS, Shin KH, Ryu N, et al. 
Inhibition by acharan sulphate of angiogenesis in experimental inflammation models. Br J Pharmacol 2002;137:441-8.

16. Jabłońska-Trypuć A, Matejczyk M, Rosochacki S. Matrix metalloproteinases (MMPs), the main extracellular matrix (ECM) enzymes in collagen degradation, as a target for anticancer drugs. J Enzyme Inhib Med Chem 2016;31:177-83.
17. Freitas-Rodríguez S, Folgueras AR, López-Otín C. The role of matrix metalloproteinases in aging: Tissue remodeling and beyond. Biochim Biophys Acta Mol Cell Res 2017;1864:2015-25.

18. Pittayapruek P, Meephansan J, Prapapan O, Komine M, Ohtsuki M. Role of matrix metalloproteinases in photoaging and photocarcinogenesis. Int J Mol Sci 2016;17:868 